\title{
Transport theory and the WKB approximation for interplanetary MHD fluctuations
}

\author{
William H. Matthaeus, Ye Zhou, ${ }^{1}$ G. P. Zank, and S. Oughton ${ }^{2}$ \\ Bartol Research Institute, University of Delaware, Newark
}

\begin{abstract}
An alternative approach, based on a multiple scale analysis, is presented in order to reconcile the traditional WKB approach to the modeling of interplanetary fluctuations in a mildly inhomogeneous large-scale flow with a more recently developed transport theory. This enables us to compare directly, at a formal level, the inherent structure of the two models. In the case of noninteracting, incompressible (Alfvén) waves, the principle difference between the two models is the presence of leading-order couplings (called "mixing effects") in the non-WKB turbulence model which are absent in a WKB development. Within the context of linearized MHD, two cases have been identified for which the leading order non-WKB "mixing term" does not vanish at zero wavelength. For these cases the WKB expansion is divergent, whereas the multiple-scale theory is well behaved. We have thus established that the WKB results are contained within the multiplescale theory, but leading order mixing effects, which are likely to have important observational consequences, can never be recovered in the WKB style expansion. Properties of the higher-order terms in each expansion are also discussed, leading to the conclusion that the non-WKB hierarchy may be applicable even when the scale separation parameter is not small.
\end{abstract}

\section{Introduction}

A central problem in solar wind physics and other areas of space physics and astrophysics is the transport of short-wavelength magnetohydrodynamic (MHD) fluctuations in a nonstationary inhomogeneous medium [Parker, 1965; Barnes, 1979, 1992; Hollweg, 1973, 1974, 1990; Barnes and Hollweg, 1974; Jacques, 1977; Heinemenn and Olbert, 1980]. In such situations, where for example WKB scaling [Jeffreys and Jeffreys, 1950; Weinberg, 1962; Bazer and Hurley, 1963; Parker, 1965; Dewar, 1970; Barnes, 1979] would be applied, the small parameter of interest is

$$
\frac{\lambda}{R} \ll 1
$$

where $\lambda$ denotes the wavelength of the fluctuation, and $R$ the scale length of variation of the mean flow. The simplest underlying description is in terms of linearized MHD theory, modified appropriately to account for the inhomogeneous background medium. A more complete

\footnotetext{
${ }^{1}$ Now at Institute for Computer Applications in Science and Engineering, Langley Research Center, Hampton, Virginia

${ }^{2}$ Now at Department of Mathematical and Computational Sciences, University of St. Andrews, Scotland, United Kingdom

Copyright 1994 by the American Geophysical Union.

Paper number 94JA02326.

0148-0227/94/94JA-02326\$05.00
}

treatment would include the nonlinear terms, but these can be separated out formally in a multiple-scales treatment, while leaving open the possibility that the nonlinearities can be modeled self-consistently.

Here we consider the linear inhomogeneous MHD problem,

$$
\frac{\partial z_{i}^{ \pm}}{\partial t}+\left(L_{x}^{ \pm}+L^{ \pm}\right) z_{i}^{ \pm}+M_{i k}^{ \pm} z_{k}^{\mp}=0,
$$

which describes the propagation of Alfvénic fluctuations in an inhomogeneous flow. Pressure terms are absent (of higher order) in these equations because the fluctuations are considered to be locally incompressible, and the pressure in that case acts only to enforce the solenoidal character of the fluctuations. The Elsässer variables are defined as

$$
\mathbf{z} \equiv \mathbf{v} \pm \frac{\mathbf{b}}{\sqrt{4 \pi \rho}}
$$

with $\mathbf{v}$ and $\mathbf{b}$ the small-scale velocity and magnetic field fluctuations. We shall denote the large-scale velocity field as $\mathbf{U}$ and the large-scale magnetic field as $\mathbf{B}$. For density $\rho$ the large-scale Alfvén speed is $\mathbf{V}_{A}=\mathbf{B} / \sqrt{4 \pi \rho}$. Finally, $L_{x}^{ \pm}, L^{ \pm}$, and the matrix $M^{ \pm}$denote linear operators that involve gradients which act both on the large-scale fields and the fluctuation (or wave) variables. Their form is given explicitly as

$$
L_{\mathbf{x}}^{ \pm}=\left(\mathbf{U} \mp \mathbf{V}_{A}\right) \cdot \nabla
$$




$$
\begin{gathered}
L^{ \pm}=\frac{1}{2} \nabla \cdot\left(\frac{1}{2} \mathbf{U} \pm \mathbf{V}_{A}\right) \\
M_{i k}^{ \pm}=\nabla_{k} U_{i} \pm \frac{1}{\sqrt{4 \pi \rho}} \nabla_{k} B_{i}-\frac{1}{2} \delta_{i k} \nabla \cdot\left(\frac{\mathbf{U}}{2} \pm \mathbf{V}_{A}\right)
\end{gathered}
$$

where $\delta_{i k}$ is the identity matrix.

It is assumed that the small-scale variables behave incompressibly. Thus only MHD Alfvén waves and not magnetosonic modes are described in this approximation. Two approaches have been developed to investigate the transport of Alvénic fluctuations described by (1): the traditional WKB method [e.g., Parker, 1965; Barnes, 1979; Hollweg, 1973; Barnes and Hollweg, 1974; Jacques, 1977] and a more recently developed multiplescales method which has some similarities to, for example, mean field electrodynamics [Zhou and Matthaeus, 1989, 1990a, b; Marsch and Tu, 1989; Tu and Marsch, 1990; Matthaeus et al., 1992].

Both approaches lead to transport equations which, although there exist many similarities, are nevertheless significantly different in many respects. Recently, there has been some discussion in the literature regarding the domain of validity of the two approaches [e.g., Hollweg, 1990; Zhou and Matthaeus, 1990a, b; Velli et al., 1989; Zhou et al., 1990; Barnes, 1992]. For example, Zhou and Matthaeus [1990b] (see also Jokipii and Kota [1989]) have argued that several situations exist in which the WKB ordering is not expected to be valid, and that the new effects appearing in the multiple-scales approach, perhaps unfortunately called "mixing effects," may be important in these cases. Specifically, within linear theory, the mixing effect should be treated in leading order when $\mathbf{k} \cdot \mathbf{V}_{A} \rightarrow 0$ or when $\mathbf{V}_{A} \rightarrow 0$, owing to the degeneracy of the two solutions to the wave dispersion relation in those limits. In contrast, Velli et al. [1989] argue that for cases of interest in models of solar wind fluctuations, these new terms are extremely small. Hollweg [1990] recognized that the WKB expansion represents a secular perturbation problem, requiring imposition of solvability conditions [e.g., Nayfeh, 1973] to regularize the expansion. Hollweg [1990] developed such an expansion for one dimensional waves, such as those considered by Heinemann and Olbert [1980], and thus his treatment was insufficiently general to address the question of the potential for important departures from the WKB expectations in the leading orders of the theory. More recently, Barnes [1992] also discussed the need to clarify this issue, in the context of WKB theory applied to magnetoacoustic waves and entropy fluctuations.

In view of the above, it is clearly important to determine under what conditions the two approaches can be reconciled. Although the description of the multiplescales mixing effects by Zhou and Matthaeus [1990b] attempted to make contact with the traditional WKB orderings, the discussion was couched in terms of transport equations for spectra or correlation functions, rather than in terms of the "primitive" linearized MHD equations. In this paper we directly address the issue of the relationship between WKB and multiple-scales treatments that include mixing effects. We develop a sin- gle multiple-scales expansion that reduces, by choice of higher-order solvability conditions, to the two separate cases of interest. This permits us to clearly identify the conditions in which the two approaches are valid, while also clarifying to a great extent the mathematical relationship that exists between the methods.

Before turning to the main developments of the paper, we describe briefly some salient features of the WKB and multiple-scales descriptions. The WKB formalism, as applied to solar wind fluctuations, is an explicitly wave description in that small-scale MHD fluctuations must satisfy the Alfvén dispersion relation. The expansion parameter is $\delta_{w k b}=1 / k R$, where $k \equiv$ wave number which satisfies the Alfvén dispersion relation, $R$ the scale length associated with the inhomogeneous flow. A requirement for accuracy of the leading order terms of the expansion is that $\delta_{w k b} \ll 1$, that is, short wavelengths with respect to the flow. It is a consequence of the wave description [Zhou and Matthaeus, $1990 \mathrm{~b}]$ that there exists no mixing, or coupling, between Alfvénic fluctuations having opposite senses of propagation, at the leading order. Mixing does, nevertheless, occur as a finite wavelength correction at higher orders of WKB theory [Heineman and Olbert, 1980]; however, this mixing is insufficient to account for the evolution of cross helicity with radial distance in the solar wind [Hollweg, 1990; Roberts et al., 1987a, b].

At leading order, WKB theory yields the equation

$$
\frac{\partial P^{ \pm}(k)}{\partial t}+L_{W K B}^{ \pm} P^{ \pm}(k)=0
$$

where we have introduced the WKB spectral transport operator

$$
\begin{aligned}
L_{W K B}^{ \pm} & \equiv\left(\mathbf{U} \mp \mathrm{V}_{A}\right) \cdot \nabla+\nabla \cdot\left(\frac{1}{2} \mathbf{U} \pm \mathbf{V}_{A}\right) \\
& =L_{\mathbf{X}}^{ \pm}+2 L^{ \pm}
\end{aligned}
$$

and $P^{ \pm}$are the reduced power spectra of the fluctuations. Note that the zero on the right-hand side of (5) illustrates that the description of strong turbulence is completely inconsistent with the WKB approach described above. In fact, for strong turbulence, a topic dealt with in the present paper in only a peripheral way, there are nonlinear couplings that appear formally when an equation such as (5) is formed. In addition, the wave dispersion relation is not obtained.

In the multiple-scales spectral transport theory [ $Z h o u$ and Matthaeus, 1989, 1990a, b; Marsch and Tu, 1989] the wave dispersion relation is not invoked, thereby introducing the possibility that "strong turbulence" can be included self-consistently. The expansion parameter is chosen to be $\delta=\lambda_{c} / R$, where $\lambda_{c}$ represents a correlation scale longer than the wavelengths of the MHD fluctuations under consideration. In this theory, mixing can now exist at the leading order; this provides the possibility of describing or explaining, at least in part, the observed radial evolution of the cross helicity in the solar wind [e.g., Roberts et al., 1987a, b; Zhou and Matthaeus, 1990a; Matthaeus et al., 1992]. The 
transport equation has the form

$$
\frac{\partial P^{ \pm}(k)}{\partial t}+L_{W K B}^{ \pm} P^{ \pm}(k)+M^{ \pm} F=N L,
$$

where $F$ denotes the spectrum of the difference between kinetic and magnetic energy and $N L$ denotes nonlinear terms responsible for local turbulence. Further equations for $\boldsymbol{F}$ and other spectral quantities, along with a closure for the nonlinear terms, are required to complete the model. The new term $M^{ \pm}$represents leading order mixing. Some solutions of these transport equations have been presented by Oughton and Matthaeus [1992] and Oughton [1993].

\section{Relation to WKB Theory: Unified Multiple-Scales Analysis}

The WKB treatment and the non-WKB multiplescales transport theories make differing predictions for leading order behavior of MHD fluctuations in a weakly inhomogeneous background flow configuration. Nonlinearities do not easily explain these discrepancies, since the mixing effect occurs in the linear transport terms. These concerns [Zhou and Matthaeus, 1990b] have been discussed in connection with the spectral transport theory, neglecting nonlinearities. It is also possible to reconcile WKB and a non-WKB mixing theory entirely in the context of the primitive linear equations for the fluctuating fields. In particular, we show in this section that both theories can be derived on the basis of a single multiple-scales development, invoking scale separation as the common element.

For a weakly inhomogeneous background flow, with fluctuations having much shorter wavelengths, we treat $\delta=\lambda_{c} / R$ as a small parameter, and introduce two length scales and two time scales to describe the evolution of the fluctuations. Corresponding to position vector $\mathbf{r}$ and time $t$ in laboratory coordinates, we define the slowly varying spatial coordinate $\mathbf{x}$ and the slowly varying time scale $\tau$ so that for small variations $d \mathbf{x}=d \mathbf{r}$ and $d \tau=d t$. These "slow" scales are associated with transport. The rapidly varying or "fast" scales, associated with the local structure and dynamics of the fluctuations, $\mathbf{x}^{\prime}$ and $\tau^{\prime}$ are defined so that $d \mathbf{x}^{\prime}=d \mathbf{r} / \delta$ and $d \tau^{\prime}=d t / \delta$. Accordingly, the position is designated by $\mathbf{r}=\mathbf{x}+\delta \mathbf{x}^{\prime}$, the time by $t=\tau+\delta \tau^{\prime}$ and derivatives are expanded as

$$
\begin{aligned}
& \nabla=\nabla \mathbf{x}+\frac{1}{\delta} \nabla_{\mathbf{x}^{\prime}}, \\
& \frac{\partial}{\partial t}=\frac{\partial}{\partial \tau}+\frac{1}{\delta} \frac{\partial}{\partial \tau^{\prime}} .
\end{aligned}
$$

Let the fluctuating Elsässer fields be expanded as

$$
\mathbf{z}^{ \pm}\left(\mathbf{x}, \mathbf{x}^{\prime}, \tau, \tau^{\prime}\right)=\mathbf{z}^{ \pm(0)}+\delta \mathbf{z}^{ \pm(1)}+\delta^{2} \mathbf{z}^{ \pm(2)}+\cdots
$$

This expansion of fields and coordinates is substituted into the linear MHD equations (1), and coefficients of like powers of $\delta$ are required to separately satisfy the equations. This give a hierarchy of equations labeled by orders of $\delta$.
The leading order $\delta^{-1}$ expansion yields

$$
\left(\partial_{\tau^{\prime}}+L_{\mathbf{x}^{\prime}}^{ \pm}\right) z_{i}^{ \pm(0)}=0
$$

where the primed operators involve derivatives with respect to the fast scale. The solution to (12) is written as $\mathbf{z}^{ \pm(0)}=\tilde{\mathbf{z}}^{ \pm(0)} e^{i S_{ \pm}}$, in which $\tilde{\mathbf{z}}^{ \pm(0)}$ is a slowly varying envelope function and $S_{ \pm}$(the eikonal or phase function) depends on both fast and slow coordinates. The fast dependence is determined by (12). The slow dependence of $S_{ \pm}$, however, is not uniquely determined, and amounts to an arbitrary phase in the fast solution. By proper choice of this slowly varying dependence of $S_{ \pm}$, one can simplify subsequent manipulations. The explicit form of the phase function that we choose is written as

$$
S_{ \pm}=\frac{1}{\delta}\left[\int_{\mathbf{r}_{0}}^{\mathbf{x}+\delta \mathbf{x}^{\prime}} \mathbf{p}^{ \pm}\left(x^{*}\right) \cdot d \mathbf{x}^{*}-\left(\tau+\delta \tau^{\prime}\right) \omega\right],
$$

with $\mathbf{r}_{0}$ a reference position. The motivation for this will become apparent when the $\mathcal{O}\left(\delta^{0}\right)$ equation is discussed. In addition, one normally treats $\delta \nabla S_{ \pm}=\nabla_{\mathbf{x}^{\prime}} S_{ \pm}=\mathbf{p}^{ \pm}$ as the local wave vector (for example in WKB theory). For consistency, this implies the auxiliary condition that

$$
\nabla \times \mathbf{p}^{ \pm}=0 .
$$

The dispersion relation

$$
D=\left(\mathbf{U} \mp \mathbf{V}_{A}\right) \cdot \mathbf{p}^{ \pm}-\boldsymbol{\omega}=0
$$

must be satisfied as a condition on the local wave vector $\mathbf{p}$ to ensure a solution to (12).

So far no assumptions regarding either of the two alternate approaches have been made. The only specialization that has been made is that the fast scale behavior of the expanded variables is written for a particular frequency component $\omega$ and a particular wave vector $\mathbf{p}$, the two being related by the dispersion relation. As is customary for linear equations, the general solution is a superposition of the amplitudes over values of $(\mathbf{p}, \omega)$. For convenience, in the following developments we will not reconstruct the full Fourier expansion.

Proceeding to the $\mathcal{O}(1)$ expansion and making use of the leading order solution yields

$$
\begin{aligned}
& \left(\partial_{\tau^{\prime}}+L_{\mathbf{X}^{\prime}}^{ \pm}\right) z_{i}^{ \pm(1)}=-e^{i S_{ \pm}}\left(\partial_{\tau}+L_{\mathbf{X}}^{ \pm}+L^{ \pm}\right) \tilde{z}_{i}^{ \pm(0)} \\
& -e^{i S_{\mp}} M_{i k}^{ \pm} \tilde{z}_{k}^{\mp(0)} \\
& -i \delta z_{p, i}^{ \pm(1)}\left(\partial_{\tau}+L_{\mathbf{X}}^{ \pm}\right) S_{\mp},
\end{aligned}
$$

where the meaning of the last term and the symbol $z_{p, i}^{ \pm(1)}$ will be presently made clear. In arriving at (16) we have made use of the property

$$
\left(\partial_{\tau}+L_{\mathbf{x}}^{ \pm}\right) S_{ \pm}=\frac{D}{\delta}=0
$$

which obtains as a consequence of our choice of slowly varying phase in (13). This important simplifying property makes it possible to treat both WKB and nonWKB hierarchies utilizing the same expansion.

Equation (16) is an inhomogeneous wave equation of the same type as the leading order $\mathcal{O}\left(\delta^{-1}\right)$ wave equa- 
tion, therefore the solution consists of a particular plus homogeneous solution

$$
\mathbf{z}^{ \pm(1)}=\mathbf{z}_{p}^{ \pm(1)}+\mathbf{z}_{h}^{ \pm(1)}
$$

The particular solution, designated as $\mathbf{z}_{p}^{ \pm(1)}$ enters into the final term of (16) and is discussed in the next section. The homogeneous solution, $\mathbf{z}_{h}^{ \pm(1)}$ has the same form (eikonal) as the zeroth-order term, hence

$$
\mathbf{z}_{h}^{ \pm(1)}=\tilde{\mathbf{z}}_{h}^{ \pm(1)} e^{i S_{ \pm}}
$$

In determining the particular solution, care must be taken to avoid secularities. This is equivalent to avoiding resonances in the inhomogeneous wave equation (16), that is, demanding that the resonant terms are identically zero. As we will show, such considerations lead to two distinct choices of solvability condition, one of which leads back to well-known classical WKB theory, while the other corresponds to the non-WKB multiplescales approach with strong mixing.

To arrive at the WKB solvability condition, assume that the inhomogeneity in the equation for $\mathbf{z}^{+(1)}$ is nonresonant with $\mathbf{z}^{-(0)}$ but is resonant with $\mathbf{z}^{+(0)}$. At this order the assumption is equivalent to $S_{+} \neq S_{-}$. Hence the only restriction on the inhomogeneous wave equation (16) is that

$$
\left(\partial_{\tau}+L_{\mathbf{X}}^{ \pm}+L^{ \pm}\right) \tilde{\mathbf{z}}^{ \pm(0)}=0 .
$$

This is the leading order WKB equation for the primitive fields $\mathbf{z}^{ \pm}$, and implies WKB transport for the energy spectra as given in (5).

The non-WKB solvability criterion is obtained through the assumption that $\mathbf{z}^{+}$and $\mathbf{z}^{-}$are nearly resonant in the sense that $S_{+} \approx S_{-}$. Hence the solvability condition for (16) is that the full right-hand side vanish; that is, the first-order corrections, $\mathbf{z}^{ \pm(1)}$, obey a homogeneous wave equation. However, we also obtain that the leading order slowly varying amplitudes obey the inhomogeneous differential equation

$$
\left(\partial_{\tau}+L_{\mathbf{x}}^{ \pm}+L^{ \pm}\right) \tilde{z}_{i}^{ \pm(0)}+\epsilon_{ \pm} M_{i k}^{ \pm} \tilde{z}_{k}^{\mp(0)}=0
$$

where $\epsilon_{ \pm}=e^{ \pm i\left(S_{-}-S_{+}\right)}$. This represents a non-WKB form of the transport equation with mixing possible at the leading order and is equivalent to (8) without the nonlinear terms. In general, the size of the mixing term depends on the magnitude of $\epsilon_{ \pm}$which in the present case is $\mathcal{O}(1)$. It is apparent that it is necessary to choose the non-WKB conditions when $S_{+} \approx S_{-}$, which is equivalent to either of the conditions $\mathbf{p}^{ \pm} \cdot \mathbf{V}_{A} \approx 0$ or $V_{A} / U \rightarrow 0$. These are two of the conditions identified previously [Zhou and Matthaeus, 1990b] for leading order mixing in linear spectral transport equations.

So far we have seen that the distinction between WKB transport and mixing type transport depends upon the choice of solvability condition on the $\mathcal{O}\left(\delta^{0}\right)$ equation. Further insights are gained by investigation of the higher-order equations and their solvability con- ditions. To avoid confusion between the two cases, we deal with them separately in the next two sections.

\section{Structure of the WKB Hierarchy}

The leading order WKB solution is of the form described after (12) and is transported at the long wavelengths according to (20). The next order corrections, in view of the solvability condition and the nonresonance of $S_{+}$and $S_{-}$, must be of the form

$$
\mathbf{z}^{ \pm(1)}=\tilde{\mathbf{z}}_{h}^{ \pm(1)} e^{i S_{ \pm}}+\tilde{\mathbf{z}}_{p}^{ \pm(1)} e^{i S_{\mp}}
$$

where $\tilde{\mathbf{z}}_{p}^{ \pm(1)}$ is the slowly varying envelope of the particular solution. Taking into account the WKB solvability condition, $\mathbf{z}_{p}^{ \pm(1)}$ must obey

$$
\begin{aligned}
\left(\partial_{\tau^{\prime}}+L_{\mathbf{X}^{\prime}}^{ \pm}\right) z_{p, i}^{ \pm(1)}=-e^{i S_{\mp}}[ & M_{i k}^{ \pm} \tilde{z}_{k}^{\mp(0)}+ \\
& \left.i \delta \tilde{z}_{p, i}^{ \pm(1)}\left(\partial_{\tau}+L_{\mathbf{X}}^{ \pm}\right) S_{\mp}\right] .
\end{aligned}
$$

The appearance of the final term in (16) and (23) is directly associated with the ansatz (22). For this form of the particular solution the wave operator and phase function are mismatched, in the term on the left-hand side, and in the final term on the right side. One can see for the fast operator that

$$
\begin{aligned}
\left(\partial_{\tau^{\prime}}+L_{\mathbf{X}^{\prime}}^{ \pm}\right) e^{i S_{\mp}} & =i e^{i S_{\mp}}\left[\mathbf{p}^{\mp} \cdot\left(\mathbf{U} \mp \mathbf{V}_{A}\right)-\omega\right] \\
& =\mp 2 i e^{i S_{\mp}} \mathbf{V}_{A} \cdot \mathbf{p}^{\mp}
\end{aligned}
$$

while, for the slow scale operator,

$$
\begin{aligned}
\left(\partial_{\tau}+L_{\mathbf{X}}^{ \pm}\right) e^{i S_{\mp}} & =i \delta^{-1} e^{i S_{\mp}}\left[\mathbf{p}^{\mp} \cdot\left(\mathbf{U} \mp \mathbf{V}_{A}\right)-\omega\right] \\
& =2 i \delta^{-1} e^{i S_{\mp}} \mathbf{V}_{A} \cdot \mathbf{p}^{\mp} .
\end{aligned}
$$

The last line in each of the above two equations follows from the first by subtracting zero in the form of the dispersion relation (15). The appearance of the factor of $\delta^{-1}$ in the second of these relations reflects the fact that the wave number corresponds to short-wavelength fluctuations when $\delta$ is small, so the phase function changes considerably when the slow scale coordinate is varied. The property embodied in (25), and in particular this factor of $\delta^{-1}$, accounts for the presence of the final term in (23), which otherwise would seem to belong in the next order equations.

The explicit form of the particular solution is readily obtained by using (22), (24), and (25) in (23) to find

$$
\tilde{z}_{p, i}^{ \pm(1)}=\frac{\left(\mathbf{M}^{ \pm} \cdot \tilde{\mathbf{z}}^{\mp(0)}\right)_{i}}{2 i\left[\omega-\mathbf{p}^{\mp} \cdot\left(\mathbf{U} \mp \mathbf{V}_{A}\right)\right]}
$$

This completely and uniquely determines $\tilde{\mathbf{z}}_{p}^{ \pm(1)}$. Again, we see that the specific choice (13) for the phase function has led to simplification. The terms involving fast and slow derivatives of the phase function contribute equally, accounting for the factor of 2 in the denominator of (26). 
As yet $\tilde{\mathbf{z}}_{h}^{ \pm(1)}$, the amplitude of the homogeneous solution at this order, is undetermined. Keeping in mind that there are a number of differences between the present treatment and that of Hollweg [1990], it appears that the analogous term in Hollweg's expansion is taken to be arbitrary and is set to zero. We will now see that, for the present formulation of the WKB hierarchy, the amplitude of this homogeneous solution is determined by higher-order solvability considerations.

At $\mathcal{O}(\delta)$ we find

$$
\begin{aligned}
\left(\partial_{\tau^{\prime}}+\right. & \left.L_{\mathbf{X}^{\prime}}^{ \pm}\right) z_{i}^{ \pm(2)}= \\
& -e^{i S_{ \pm}}\left[\left(\partial_{\tau}+L_{\mathbf{X}}^{ \pm}+L^{ \pm}\right) \tilde{z}_{h, i}^{ \pm(1)}+M_{i k}^{ \pm} \tilde{z}_{p, k}^{\mp(1)}\right] \\
& -e^{i S_{\mp}}\left[\left(\partial_{\tau}+L_{\mathbf{X}}^{ \pm}+L^{ \pm}\right) \tilde{z}_{p, i}^{ \pm(1)}+M_{i k}^{ \pm} \tilde{z}_{h, k}^{\mp(1)}\right] \\
& -i \delta z_{p, i}^{ \pm(2)}\left(\partial_{\tau}+L_{\mathbf{X}}^{ \pm}\right) S_{\mp},
\end{aligned}
$$

where the last term on the right-hand side anticipates a particular solution with mismatched phase function.

Viewed as an equation that determines the fast scale behavior of $\tilde{\mathbf{z}}^{ \pm(2)}$, we note the following structure of (27). First, the WKB solvability condition requires that the resonant term on the right-hand side vanish separately, so that secularities in the fast scale quantities on the left-hand side are avoided. Thus we require that

$$
\left(\partial_{\tau}+L_{\mathbf{X}}^{ \pm}+L^{ \pm}\right) \tilde{z}_{h, i}^{ \pm(1)}+M_{i k}^{ \pm} \tilde{z}_{p, k}^{\mp(1)}=0 .
$$

Equation (28) provides a connection between the first-order homogeneous and particular solutions, relating their slow scale variations. Technically, (28) is an inhomogeneous equation for $\tilde{\mathbf{z}}_{h}^{ \pm(1)}$, and we could argue that it involves a further decomposition into a homogeneous and particular solution, referring now to the slow scale behavior. However, such a homogeneous solution to (28) would obey the analogue of (20) at the slow scales and the analogue of (12) at the fast scales. Thus, without loss of generality, this type of contribution to $\tilde{\mathbf{z}}_{h}^{ \pm(1)}$ can be grouped with the leading order solution, $\tilde{\mathbf{z}}^{ \pm(0)}$, since it obeys the same equations. The only solution to the solvability condition equation (28) that we need to consider is then the particular solution with source $-M_{i k}^{ \pm} \tilde{z}_{p k}^{\mp(1)}$. This linear equation is formally solved by a Green's function, giving an explicit expression for $\tilde{\mathbf{z}}_{h}^{ \pm(1)}$ in terms of $\tilde{\mathbf{z}}_{p}^{\mp(1)}$,

$$
\tilde{z}_{h, i}^{ \pm(1)}=\int d \mathbf{y} \int d \eta G_{i l}(\mathbf{x}-\mathbf{y}, t-\eta) M_{l m}^{ \pm} \tilde{z}_{p, m}^{\mp(1)}(\mathbf{y}, \eta)
$$

where $\mathbf{y}$ and $\eta$ are slow coordinates of integration, and the source function can also be rewritten in terms of $\tilde{\mathbf{z}}^{ \pm(0)}$ if desired, through (26).

Having dealt with the solvability condition at this order, (27) reduces to an inhomogeneous equation, the solutions to which will consist of a homogeneous and a particular solution, $\mathbf{z}^{ \pm(2)}=\mathbf{z}_{h}^{ \pm(2)}+\mathbf{z}_{p}^{ \pm(2)}$. The homogeneous part satisfies the by now familiar wave equation,

$$
\left(\partial_{\tau^{\prime}}+L_{\mathbf{X}^{\prime}}^{ \pm}\right) z_{i}^{ \pm(2)}=0
$$

so that the solution takes the form $\mathbf{z}_{h}^{ \pm(2)}=\tilde{\mathbf{z}}_{h}^{ \pm(2)} e^{i S_{ \pm}}$ Next, the particular solution to (27) is written, in analogy to (26) and (29), and taking into account both (24) and (25), as

$$
\tilde{z}_{p, i}^{ \pm(2)}=\frac{\left(\mathbf{M}^{ \pm} \cdot \tilde{\mathbf{z}}_{h}^{\mp(1)}\right)_{i}+\left(\partial_{\tau}+L_{\mathbf{x}}^{ \pm}+L^{ \pm}\right) \tilde{z}_{p, i}^{ \pm(1)}}{2 i\left[\omega-\mathbf{p}^{\mp} \cdot\left(\mathbf{U} \mp \mathbf{V}_{A}\right)\right]}
$$

Thus the full solution to (27) takes the form $\mathbf{z}^{ \pm(2)}=$ $\tilde{\mathbf{z}}_{h}^{ \pm(2)} e^{i S_{ \pm}}+\tilde{\mathbf{z}}_{p}^{ \pm(2)} e^{i S_{\mp}}$, with the fast behavior of the homogeneous part determined by (30) while both the slow and fast behavior of the particular solution is determined by (31).

A structure similar to what we have just seen occurs at all higher orders in the WKB hierarchy. In fact, for all orders $\alpha \geq 1$, the solutions can be written in terms of the phase functions as homogeneous and particular parts in the familiar form $\mathbf{z}^{ \pm(\alpha)}=\tilde{\mathbf{z}}_{h}^{ \pm(\alpha)} e^{i S_{ \pm}}+$ $\tilde{\mathbf{z}}_{p}^{ \pm(\alpha)} e^{i S_{\mp}}$, with the homogeneous envelope function determined (as a consequence of a solvability condition) by the formal solution, in analogy to (29), to the equation

$$
\left(\partial_{\tau}+L_{\mathbf{x}}^{ \pm}+L^{ \pm}\right) \tilde{z}_{h, i}^{ \pm(\alpha)}+M_{i k}^{ \pm} \tilde{z}_{p, k}^{\mp(\alpha)}=0,
$$

in which we view the particular solution as a given source function. According to the argument given in the previous paragraph, this Green's function solution for $\tilde{z}_{h, i}^{ \pm(\alpha)}$ can be chosen to be the entire solution to the homogeneous problem at $\mathcal{O}(\alpha)$, by proper choice of the definition of the leading order solutions. Similarly, for all orders $\alpha>1$ the particular solution envelope functions are given in terms of the prior order solutions according to

$$
\tilde{z}_{p, i}^{ \pm(\alpha)}=\frac{\left(\mathbf{M}^{ \pm} \cdot \tilde{\mathbf{z}}_{h}^{\mp(\alpha-1)}\right)_{i}+\left(\partial_{\tau}+L_{\mathbf{x}}^{ \pm}+L^{ \pm}\right) \tilde{z}_{p, i}^{ \pm(\alpha-1)}}{2 i\left[\omega-\mathbf{p}^{\mp} \cdot\left(\mathbf{U} \mp \mathbf{V}_{A}\right)\right]}
$$

The assembly of the entire formal WKB hierarchy is now complete, and a full solution can be obtained as follows. First, we select the specific form of the operators $L_{\mathbf{X}}^{ \pm}, L^{ \pm}$and $M_{i j}^{ \pm}$, which depend upon the fixed background flow and magnetic field. At the inner boundary, say the Alfvénic critical point or some other surface, boundary data are chosen for the field $\mathbf{z}^{ \pm(0)}$. Without loss of generality, all other fluctuation fields in the expansion are selected to have value zero at the inner boundary. The leading order fields evolve at the long wavelengths and low frequencies in accordance with (28), with fast scale behavior determined by (12)-(15). The first correction consists of a particular solution determined by (31), and homogeneous part whose fast evolution obeys the usual wave equation (the homogeneous form of (27)), with the slow envelope determined by (28). All higher orders are determined by use of (33) to obtain the particular solution from the previous order, and then employing (32) to determine the envelope of the homogeneous part. 
It remains to discuss the conditions for accuracy, and possible convergence, of the series solution obtained through the WKB procedure specified above. However, we defer consideration of WKB convergence properties momentarily in order to address the structure of the higher-order terms associated with use of the non-WKB solvability condition.

\section{Structure of the non-WKB Hierarchy}

We return now to the hierarchy of equations implied by the selection of the solvability condition (21) in the $\mathcal{O}\left(\delta^{0}\right)$ transport equation (16). This choice implies that $\mathbf{z}^{ \pm(1)}$ obeys a sourceless wave equation, and that, therefore the particular solution in (18) vanishes. This property considerably simplifies the $\mathcal{O}(\delta)$ equations, which reduce to

$$
\begin{aligned}
\left(\partial_{\tau^{\prime}}+L_{\mathbf{X}^{\prime}}^{ \pm}\right) z_{i}^{ \pm(2)}= & -e^{i S_{ \pm}}\left(\partial_{\tau}+L_{\mathbf{X}}^{ \pm}+L^{ \pm}\right) \tilde{z}_{i}^{ \pm(1)} \\
& -e^{i S_{\mp}} M_{i k}^{ \pm} \tilde{z}_{k}^{\mp(1)}
\end{aligned}
$$

In the spirit of the argument leading to (21), we choose the solvability condition to be the vanishing of the entire right hand side of (34), thus ignoring possible nonresonance of source terms with distinct phase factors. This reduces (34) to a sourceless wave equation for the fast $\mathcal{O}\left(\delta^{2}\right)$ solution, supplemented by the slow envelope equation

$$
\left(\partial_{\tau}+L_{\mathbf{x}}^{ \pm}+L^{ \pm}\right) \tilde{z}_{i}^{ \pm(1)}+\epsilon_{ \pm} M_{i k}^{ \pm} \tilde{z}_{k}^{\mp(1)}=0
$$

with $\epsilon_{ \pm}$having the definition given following (20). The envelope equation for $\tilde{\mathbf{z}}^{ \pm(1)}$ is identical in form to $(20)$, the analogous equation obeyed by $\tilde{\mathbf{z}}^{ \pm(0)}$.

It is immediately apparent that all higher orders of expansion generated by the non-WKB solvability condition will share several properties. First, all of the fast space/timescale wave equations are homogeneous, so there will be no particular solutions, only homogeneous solutions. Second, all solvability conditions obtained in this fashion will have the same structure, given by (21) and (35).

These simple but powerful properties of the nonWKB solvability approach permit the entire problem to be recast in simpler terms. We may resum the entire series given in (11), using the eikonal ansatz for the form of the solution,

$$
\mathbf{z}^{ \pm}=\tilde{\mathbf{z}}^{ \pm} e^{i S_{ \pm}}
$$

The entire perturbative solution can then be directly summed to all orders and is found to obey the pair of equations

$$
\begin{aligned}
\frac{\tilde{z}_{i}^{ \pm}}{\delta}\left(\partial_{\tau^{\prime}}+L_{\mathbf{x}^{\prime}}^{ \pm}\right) S_{ \pm} & =0 \\
\left(\partial_{\tau}+L_{\mathbf{x}}^{ \pm}+L^{ \pm}\right) \tilde{z}_{i}^{ \pm}+\epsilon_{ \pm} M_{i k}^{ \pm} \tilde{z}_{k}^{\mp} & =0 .
\end{aligned}
$$

This result, obtained here using only the eikonal ansatz and the scale separation assumption, is evidently equiv- alent to the full non-WKB hierarchy. This is discussed further below.

\section{Convergence and Accuracy of WKB and non-WKB Expansions}

Our motivation originates in applications, such as the solar wind, where the scale separation parameter $\delta$ is small, that is, the fluctuations have wavelengths small compared to characteristic background scales. Consequently, we assumed $\delta \ll 1$ in both WKB and nonWKB treatments, but in the non-WKB hierarchy no assumptions were made concerning $\epsilon_{ \pm}$. When the conditions that motivated the non-WKB solvability condition are satisfied, $\epsilon_{ \pm} \approx 1$ and this approach must be preferred to the WKB approach, which is expected to fail in that case. In the event that the WKB solvability condition is permissible, $\epsilon_{ \pm}$is rapidly varying on the fast scales, and has the effect of a parameter with magnitude $\ll 1$. In that case the couplings that enforce interactions between the $z^{+}$and $z^{-}$are weak, that is, there is little mixing. However, with regard to the nonWKB formalism, this means only that a small term has been kept in each order, one that might have been relegated to higher order in $\delta$. This will not invalidate the procedure. In particular, the series still resums to give (37)-(38), although the mixing effects remain small.

The fact that all orders of the non-WKB expansion obey the same equations suggests that even $\delta<1$ may not be a formal requirement for accuracy and convergence. Suppose we consider the original lincarized MHD equations (1) and assume only that the solutions we seek take the eikonal form

$$
\mathbf{z}^{ \pm}=\tilde{\mathbf{z}}^{ \pm} e^{i S_{ \pm}}
$$

Substitution into (1) produces the single equation

$$
\begin{aligned}
& \frac{i \tilde{z}_{i}^{ \pm}}{\delta}\left[\left(\partial_{\tau^{\prime}}+L_{\mathbf{X}^{\prime}}^{ \pm}\right) S_{ \pm}\right]+ \\
& \quad\left(\partial_{\tau}+L_{\mathbf{x}}^{ \pm}+L^{ \pm}\right) \tilde{z}_{i}^{ \pm}+\epsilon_{ \pm} M_{i k}^{ \pm} \tilde{z}_{k}^{\mp}=0 .
\end{aligned}
$$

Now let the phase function $S_{ \pm}$satisfy the familiar conditions (13)-(15). This causes the term multiplying $\delta^{-1}$ to vanish separately, a statement equivalent to (37). The remainder of (40) reduces to the non-WKB envelope equation involving mixing of the \pm fluctuations, with intrinsic strength $\epsilon_{ \pm}$, a relation identical to (38). Consequently, the resummed non-WKB hierarchy is seen to be fully equivalent to the full linearized MHD equations. The approximation being made is simply that the eikonal relations (13)-(15) permit the fast part of the wave operator to separately vanish. This will be legitimate when the scale separation condition $\delta \ll 1$ is met. However, the general approach outlined is valid (and exact) whenever a formulation for $S_{ \pm}$can be found that obeys (37).

In this framework it appears that the non-WKB formalism is perturbative only to the extent that errors of some order in $\delta$ might exist in the underlying eikonal properties. Naturally, one need also keep in mind that 
general formulations of the linearized MHD equations might themselves give rise to solutions that become unbounded in space or time. However, the non-WKB formalism appears to be as robust as the linearized MHD equations themselves, provided that accurate expressions for the eikonal (13) can be found. In particular, the above argument suggests that the non-WKB formalism, with $S_{ \pm}$obeying (37) exactly, is always convergent, whenever the solutions to (1) exist. In applications to the solar wind fluctuation problem, in the short-wavelength limit, the non-WKB formalism becomes identical to linearized MHD.

Consideration of the general conditions for convergence of the WKB hierarchy is more difficult. One needs to determine the general conditions for the iteration implied by (33) and (32) to remain bounded. Rather than pursue this, it seems more useful to characterize conditions necessary for the accuracy of the first several terms in the WKB hierarchy. For accuracy, we would require, for example, that $\left|\tilde{z}_{i}^{ \pm(\alpha+1)}\right| \leq\left|\tilde{z}_{i}^{ \pm(\alpha)}\right|$, and other relations such as $\left|\tilde{z}_{p, i}^{\mp(1)}\right| \leq\left|\tilde{z}_{i}^{ \pm(0)}\right|$ and $\left|\tilde{z}_{h, i}^{ \pm(1)}\right| \leq\left|\tilde{z}_{i}^{ \pm(0)}\right|$. We proceed to discuss the extent to which these might be valid using estimates of the terms in (33) and (32).

For simplicity, we estimate the effect of the matrix $M_{i k}^{ \pm}$by a factor $M^{ \pm} \approx 2\left(U \pm V_{A}\right) / R$, where $R$ is the local heliocentric distance coordinate. (This is appropriate for a spherical expansion at constant speed $U$, see, for example, Zhou and Matthaeus [1990a].) In view of (25) we may write $\pm 2 \mathbf{V}_{A} \cdot \mathbf{p}^{\mp}= \pm 2 V_{A} p^{\mp} \cos \theta$, where $\theta$ is the angle between the local wavevector and the large scale Alfvén speed $\mathbf{V}_{A}$. Then (26), along with the accuracy condition $\left|\tilde{z}_{p, i}^{ \pm(1)}\right| \leq\left|\tilde{z}_{i}^{ \pm(0)}\right|$, suggests the requirement that

$$
\frac{2\left(U \pm V_{A}\right)}{R} \frac{1}{4 V_{A} p^{\mp} \cos \theta} \leq 1
$$

or

$$
\frac{1}{p^{\mp} R} \leq \frac{2 V_{A} \cos \theta}{U \pm V_{A}},
$$

in addition to the scale separation condition

$$
\frac{1}{p^{\mp} R} \leq 1 .
$$

Clearly, the first of these conditions is more restrictive than the second for the solar wind, in which $V_{A}<U$. In addition, we can estimate the terms in the slow spatial transport operator on the left side of (28) as of order $\approx\left(U \pm V_{A}\right) / R$. Thus, because the homogeneous and particular solutions are connected by (28) and (32), they are expected to be of the same order.

In this elementary assessment, one sees immediately that the WKB procedure will lose accuracy (and possibly convergence as well) when $\cos \theta \rightarrow 0$, corresponding to a "two-dimensional" fluctuation geometry, with wave vectors perpendicular to the local large-scale magnetic field. It also becomes inaccurate when $V_{A} / U \rightarrow 0$, corresponding to negligible frequency difference between outward $(+)$ and inward $(-)$ propagating waves [Holl- weg, 1990]. These conditions cause $S_{+} \approx S_{-}$, distinguishing the cases in which we argued earlier that the non-WKB solvability conditions need to be enforced. Accordingly, the rationale for utilizing the WKB solvability condition fails, and its use can produce unbounded results. In these situations, the non-WKB hierarchy is preferable and can be expected to produce more accurate results. In the context of spectral transport theories the same cases were identified as requiring use of a transport theory with the possibility of strong mixing between $\mathbf{z}^{+}$and $\mathbf{z}^{-}$[Zhou and Matthaeus, $1990 \mathrm{~b}]$.

\section{Numerical Examples}

In order to illustrate the substantial differences between approximate solutions to (1) resulting from the imposition of either the nonresonant or the resonant solvability conditions, we now present some pertinent numerical results. We present solutions to the spectral form of the transport equations associated with the leading order slowly varying terms in the WKB and non-WKB hierarchies, for example, (5) and (8), rather than the fluctuations themselves, since this is often the application of interest [see $T u$ et al., 1984; $T u, 1988$; Marsch and Tu, 1989; Tu and Marsch, 1990; Zhou and Matthaeus, 1990a]. As the spectral transport equations are derived directly from the equations for the fluctuations, there is a clear correspondence between the two forms of the solutions. The details of the derivation and structure of the spectral transport equations are not relevant here, and we refer readers to previous work for further discussion [Zhou and Matthaeus, 1990a; Oughton and Matthaeus, 1992; Oughlon, 1993]. What is important for the present illustrations is that the spectral equations admit mixing effects in the same limits in which they are expected for the primitive scaleseparated equations. Thus, the influence of geometry (slab versus two dimensional), magnitude of wave number, and magnitude of Alfvén speed can be investigated in a straightforward fashion by computing solutions to the spectral transport equations.

Chebyshev pseudospectral methods were used to compute solutions to (5) and (8) with the nonlinear terms neglected. The latter (non-WKB) case requires additional equations for closure since fields other than the inward $\left(P^{-}\right)$and outward $\left(P^{+}\right)$energies are involved.

Since the full three-dimensional, time-dependent solution of the problem is currently out of reach, we restrict ourselves to the one-dimensional system in which all spectral quantities depend solely upon the heliocentric radial coordinate. With a view toward solar wind applications the large-scale velocity $\mathbf{U}$ is taken to be constant and radially outward; $\mathbf{B}_{0}$ is the standard Parker spiral; and $\rho \propto R^{-2}$. We make the further approximation, not crucial for the present purpose, that the small-scale turbulence is, and remains, either isotropic, slab, or two-dimensional (2D). This causes the mixing operators to reduce to algebraic functions of radial distance so that the equations are then relatively 
straightforward to solve [Zhou and Matthaeus, 1990a; Oughton and Matthaeus, 1992; Oughton, 1993].

The boundary conditions imposed are those appropriate for supersonic, super-Alfvénic flow which begins at the Alfvén critical radius (where $V_{A r}=U$ ). More specifically we have purely outward type fluctuations, which requires that the inward energy and the energy difference, $F$, are zero at this distance. (Hollweg [1990] has remarked that a small admixture of inward waves may be required for consistency with higher order terms in the expansions, but we neglect this effect here.) Since our equations are linear, the actual value of $P^{+}>0$ is immaterial. Steady state solutions to these transport equations, for the special case $V_{A} / U=0$ case show marked departures from standard WKB solutions [Zhou and Matthaeus, 1990a], as is consistent with discussions in earlier sections above.

The numerical solutions will be compared using a single diagnostic quantity, the normalized cross helicity $\sigma_{c}=\left(P^{+}-P^{-}\right) /\left(P^{+}+P^{-}\right)$. This quantity is bounded, the extremal values corresponding to purely outward propagating Alfvén waves $\left(\sigma_{c}=1\right)$ and purely inward propagating ones $\left(\sigma_{c}=-1\right)$. Intermediate values correspond to a superposition of $\mathbf{z}^{+}$and $\mathbf{z}^{-}$, irrespective of whether or not these fluctuations are propagating, interacting, or associated with mixing effects. This diagnostic is sensitive to the accuracy of the leading order WKB solution, which predicts, for the chosen boundary data, that $\sigma_{c}=+1$ for all heliocentric distances $R$, corresponding to purely outward propagating waves at all distances.

Figure 1 shows plots of $\sigma_{c}$ versus heliocentric distance for the case of isotropic turbulence with an inertial range spectral slope of $-5 / 3$. It is important to note that for isotropic fluctuations [Oughton and Matthaeus, 1992; Oughton, 1993] the equation for the evolution of the energy difference spectrum (cf. (21)) lacks an oscillatory term $\sim \mathbf{k} \cdot \mathbf{V}_{A}$ that would act to enforce WKB behavior. Since the "WKB enforcing" terms are the only wave number dependent terms in the linear the-

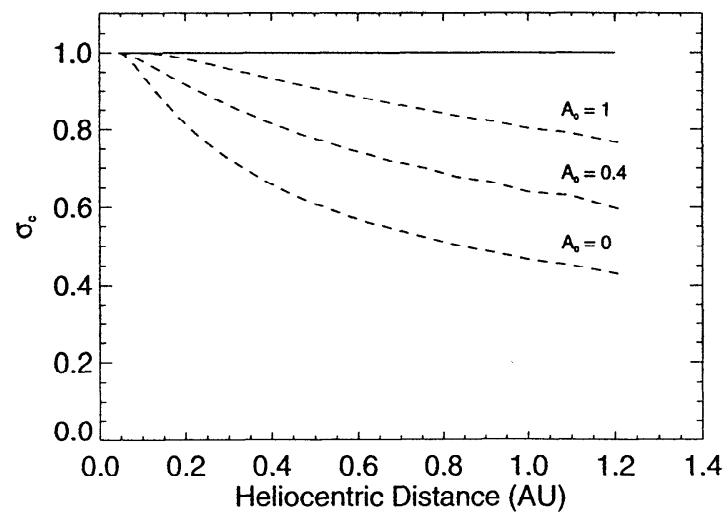

Figure 1. Normalized cross helicity as a function of heliocentric distance. The dashed curves are the leading order solutions for the non-WKB hierarchy when the small-scale turbulence is isotropic, for varying $A_{0}$, the ratio of Alfvén speed to flow speed at the inner boundary. The leading order WKB solution is shown as the solid line.

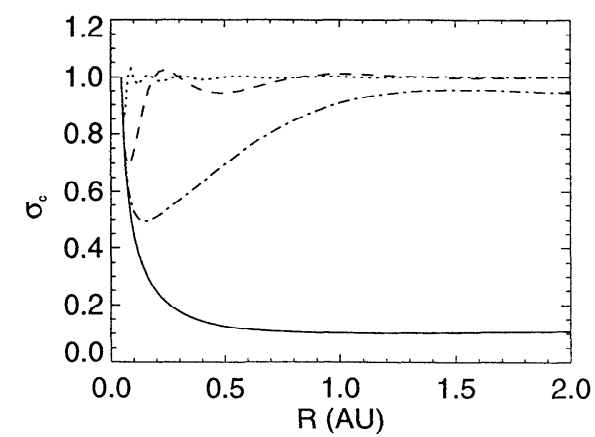

Figure 2. Leading order non-WKB solutions for the case of slab turbulence at various wave numbers. The solid curve is the normalized cross helicity for $k=0$ (and also for the 2D geometry); moving up the figure the broken curves are respectively for $k=1$ (dash-dot), 2 (dash), and 5 (dot).

ory, the isotropic results are independent of the wave number of the fluctuations. Results for three values of $A_{0}=V_{A r}\left(R=R_{0}\right) / U$, are shown, namely $0,0.4$ and 1 , along with the standard (leading order) WKB theory $\left(\sigma_{c}=1\right)$. As $A_{0}$ decreases, the solutions show greater departures from the WKB result, in agreement with the properties of the WKB and non-WKB solutions discussed in section .

For slab turbulence (i.e., transverse fluctuations with wave vector $\mathbf{k}$ parallel to $\mathbf{B}_{0}$ ) the WKB enforcing terms alluded to above are present, and convergence to WKB results is expected at short wavelength. Slab results with $A_{0}=1$ are shown in Figure 2. Solutions for the (nonzero) values of $k$ depicted in Figure 2 are such that $1 / k L$, varies from $1 / 10$ to $1 / 50$, where $L \approx 0.5 \mathrm{AU}$ is a characteristic length scale for the background fields. In addition, a solution for $k=0$ is shown.

The extremely rapid convergence to WKB-like solutions as $k$ increases away from zero is evident. Well before $k$ reaches a value corresponding to correlation lengths typically observed at $1 \mathrm{AU}$ in the solar wind $(\approx 1 / 50 \mathrm{AU})$, the solutions are essentially equivalent to the WKB results.

As a final point in this section, note that the solutions for 2D small-scale turbulence are always of the nonWKB form; that is, mixing is important. Formally, the $2 \mathrm{D}$ result for the model numerical problem is equivalent to the $k=0$ slab result [Oughton, 1993], which departs substantially from the WKB result.

\section{Discussion}

We have shown that the WKB and non-WKB hierarchies can be obtained from a single asymptotic expansion, distinguished according to the imposed solvability conditions. Application of the non-WKB solvability condition in each order regardless of whether $\mathbf{z}^{+}$and $\mathbf{z}^{-}$are resonant or not yields homogeneous wave equations at all orders. Hence every order of the expansion takes on the same form, leading to a valid resummation of the asymptotic series and thus giving $\mathbf{z}^{ \pm}$. By contrast, the WKB solvability condition introduces a much more complex hierarchy of perturbed equations whose 
solutions consist always of homogeneous and particular parts. Consequently, resumming the asymptotic series proves extremely difficult. The solvability conditions for the non-WKB approach prove less restrictive, and therefore the regime of convergence for the WKB theory is correspondingly smaller.

It is apparent that it is necessary to choose the nonWKB conditions when $S_{+} \approx S_{-}$, which is equivalent to either of the conditions

$$
\begin{aligned}
\mathbf{p}^{ \pm} \cdot \mathbf{V}_{A} & \approx 0 \\
V_{A} / U & \rightarrow 0
\end{aligned}
$$

$U$ the mean flow velocity. These are two of the conditions identified previously by [Zhou and Matthaeus, $1990 \mathrm{a}, \mathrm{b}]$ in their development of the non-WKB transport theory for spectral quantities.

Where applicable, the lowest-order WKB theory is substantially simpler, since it allows for the possibility, in leading order of following each of the fluctuation types independently. For example, in the solar wind, WKB theory allows the evolution of "outward traveling" waves to be computed with no reference whatsoever to an admixture of inward waves. In contrast, the non-WKB formalism always requires that equations for both species of fluctuations be followed. While this implies that the leading order equations are more complex than in WKB theory, the higher-order equations are simpler, since no particular solutions appear. Moreover, the non-WKB theory collapses to the WKB theory when the latter is valid, as suggested by the analysis and demonstrated by our numerical examples.

The reason for the greater complexity of the WKB heirarchy at orders beyond the first is easy to understand. The WKB strategy is to simplify the leading order description, which is accomplished by simultaneously exploiting the separation of length scales and the rapid phase interference of waves propagating in opposite directions. In cases where this works it completely decouples the dynamics of inward and outward traveling waves. However, in general the effects of phase interference and scale separation are physically distinct, and are associated with two independent small parameters. The price paid for the leading order WKB simplification is that higher-order corrections are more complicated and become large in certain limits. The nonWKB heirarchy makes no assumption about phase separation, and exploits only one effect, that of scale separation. Consequently, the non-WKB procedure has the same complexity at all orders, being more complex than WKB in leading order, but subsequently more simple.

The numerics show that isotropic fluctuations (with the symmetry maintained by unspecified effects) display sensitivity to the strength of the Alfvén speed $V_{A}$ relative to the radial flow speed $U$, with weaker $V_{A}$ cases departing more from WKB expectations. Slab fluctuation geometry shows rapid convergence to the WKB result as the wavelength is decreased. It appears that the entire powerlaw inertial range of wave numbers in the solar wind (as measured at $1 \mathrm{AU}$ ) lies within the range of accuracy of WKB theory, if these fluctuations are assumed to be of slab geometry and described by linearized MHD. In contrast, 2D fluctuations never approach WKB theory, for any wavelength or Alfvén speed. (See, e.g., Matthaeus et al. [1990] for an interpretation of solar wind observations as 2D fluctuations.) The numerical results are completely consistent with the discussion in section regarding the applicability of the WKB approach for these cases.

Under general circumstances, non-WKB theory provides for the possibility of mixing, a perhaps unfortunate terminology for the coupling of oppositely-traveling Alfvénic fluctuations at leading order. Suggestions have been made [e.g., Zhou and Matthaeus, 1990a] that this may account for the observed evolution of cross-helicity in the solar wind [Roberts et al., 1987a, b]. The abovedescribed unified treatment of mixing and standard WKB theories (in which mixing is at higher order) may help to clarify the nature of this effect and the relationship between spectral transport theories of these two types [e.g., Tu et al., 1984; Marsch and Tu, 1989; Tu and Marsch, 1990; Zhou and Matthaeus, 1990a].

While the present paper has dealt exclusively with linear MHD, nonlinear effects can be incorporated within the non-WKB framework in a self-consistent manner. However, this is not the case for classical WKB theory, since strong nonlinear effects will involve substantial departures from the frequency/wavenumber relation embodied in the dispersion relation, which is required for validity of the $\mathrm{WKB}$ ordering. (Note that Tu et al. [1984] have argued that in certain limits, namely weak turbulence, the WKB transport operator may be compatible with nonlinear effects.) We expect that the nonWKB treatment is preferable and quite likely required in any transport theory involving strong MHD turbulence.

Finally, in the present discussion we have dealt mainly with the case of fluctuations with wavelength small compared to background inhomogeneities. However, our analysis has suggested that the non-WKB formulation of linear inhornogeneous MHD may remain accurate whenever a suitable eikonal relation can be defined, even if the scale separation parameter is not small. This indicates that the non-WKB formalism may find application in situations with rapidly varying but organized mean fields, such as MHD shock problems.

Acknowledgments. This work is supported by NASA through the Space Physics Theory Program (grant NAG5-1573) at Bartol. G.P.Z. is partially supported by the NSF NYI program (grant ATM-9357861) at Bartol. S.O. is presently supported by PPARC st St. Andrews. Computational support provided by the NSF San Diego supercomputer center.

The editor thanks D. A. Roberts and Z. E. Musielak for their asistance in evaluating this paper.

\section{References}

Barnes, A., Hydromagnetic waves and turbulence in the solar wind. in Solar System Plasma Physics, vol. I, edited by E. N. Parker, C. F. Kennel, and L. J. Lanzerotti, p. 251, North-Holland, New York, 1979.

Barnes, A., Theory of magnetohydrodynamic waves: The WKB approximation revisited, J. Geophys. Res., 97, 12,105 1992. 
Barnes, A., and J. Hollweg, Large amplitude hydromagnetic waves, J. Geophys. Res., 79, 2302, 1974.

Bazer, J., and J. Hurley, Geometrical hydromagnetics, $J$. Geophys. Res., 68, 147, 1963.

Dewar, R. L., Interaction between hydromagnetic waves and a time-dependent, inhomogeneous medium, Phys. Fluids, 13, 2710, 1970.

Heinemann, M., and S. Olbert, Non-WKB Alfvén waves in the solar wind, J. Geophys. Res., 85, 1311, 1980.

Hollweg, J. V., Alfven waves in the solar wind: Wave pressure, Poynting flux, and angular momentum, J. Geophys. Res., 78, 3643, 1973.

Hollweg, J. V., Transverse Alfven waves in the solar wind: Arbitrary $\mathbf{k}, \mathbf{V}_{0}, \mathbf{B}_{0}$ and $|\delta \mathbf{B}|, J$. Geophys. Res., 79, 1539, 1974.

Hollweg, J. V., On WKB expansions for Alfvén waves in the solar wind, J. Geophys. Res., 95, 14,873, 1990.

Jacques, S., Momentum and energy transport by waves in the solar atmosphere and solar wind, Astrophys. J., 215, $942,1977$.

Jeffreys, H., and B. S. Jeffreys, Methods of Mathematical Physics, Cambridge University Press, New York, 1950.

Jokipii, J. R., and J. Kota, The polar heliospheric magnetic field, Geophys. Res. Lett., 16, 1, 1989.

Marsch, E., and C.-Y. Tu, Dynamics of correlation functions with Elsässer variables for inhomogeneous mhd turbulence, J. Plasma Phys., 41, 479, 1989.

Marsch, E., and C.-Y. Tu, Modeling results on spatial transport and spectral transfer of solar wind Alfvénic turbulence, J. Geophys. Res., 98, 21,045, 1993.

Matthaeus, W. H., M. L. Goldstein, and D. A. Roberts, Evidence for the presence of quasi-two dimensional nearly incompressible fluctuations in the solar wind, J. Geophys. Res., 95, 20673, 1990.

Matthaeus, W. H., Y. Zhou, S. Oughton, and G. P. Zank, Weakly inhomogeneous mhd turbulence and transport of solar wind fluctuations, in Proceedings of Solar Wind 7, COSPAR Colloq. Ser., vol. 3, edited by E. Marsch and R. Schwenn, p. 511, Pergamon, New York, 1993.

Nayfeh, A., Perturbation Methods, John Wiley, New York, 1973.

Oughton, S., Transport of solar wind fluctuations: A turbulence approach, Ph.D. thesis, Univ. of Del., Newark, 1993.

Oughton, S. and W. H. Matthaeus. Evolution of solar wind fluctuations and the influence of turbulent 'mixing,' in Proceedings of Solar Wind 7, COSPAR Colloq. Ser., vol. 3, edited by E. Marsch and R. Schwenn, p. 523, Pergamon, New York, 1993.

Parker, E. N., Dynamical theory of the solar wind, Space Sci. Rev., 4, 666, 1965.

Roberts, D. A., L. W. Klein, M. L. Goldstein, and W. H. Matthaeus, The nature and evolution of magnetohydro- dynamic fluctuations in the solar wind: Voyager observations, J. Geophys. Res., 92, 11 021, 1987a.

Roberts, D. A., M. L. Goldstein, L. W. Klein, and W. H. Matthaeus, Origin and evolution of fluctuations in the solar wind: Helios observations and Helios-Voyager comparisons, J. Geophys. Res., 92, 12,023, 1987b.

Tu, C.-Y., The damping of the interplanetary Alfvenic fluctuations and the heating of the solar wind, J. Geophys. Res., 93, 7, 1988.

Tu, C.-Y., and E. Marsch, Transfer equations for spectral densities of inhomogeneous mhd turbulence, J. Plasma Phys., 44, 103, 1990.

C.-Y. Tu and E. Marsch, A model of solar wind fluctuations with two components: Alfvén waves and convective structures, J. Geophys. Res., 98, 1257, 1993.

Tu, C.-Y., Z. Pu, and F. Wei, The power spectrum of interplanetary Alfven wave fluctuations: Derivation of the governing equation and its solutions, J. Geophys. Res., 89, 9695, 1984.

Velli, M., R. Grappin, and A. Mangeney, Turbulent cascade of incompressible unidirectional Alfvén waves in the interplanetary medium, Phys. Rev. Lett., 63, 1807, 1989.

Weinberg, S., Eikonal method in magnetohydrodynamics, Phys. Rev., 126, 1899, 1962.

Zhou, Y., and W. H. Matthaeus, Non-WKB evolution of solar wind fluctuations: A turbulence modeling approach, Geophys. Res. Lett., 16, 755, 1989.

Zhou, Y., and W. H. Matthaeus, Transport and turbulence modeling of solar wind fluctuations, J. Geophys. Res., 95, 10,291, 1990a.

Zhou, Y., and W. H. Matthaeus, Remarks on transport theories of interplanetary fluctuations, J. Geophys. Res., 95, $14,863,1990 \mathrm{~b}$.

Zhou, Y., W. H. Matthaeus, D. A. Roberts, and M. L. Goldstein, Physical consistency in modeling interplanetary magnetohydrodynamic fluctuations, Phys. Rev. Lett., $64,2591,1990$.

W. H. Matthaeus and G. P. Zank, Bartol Research Institute, University of Delaware, Newark, DE 19716 (e-mail: yswhm@bartol.udel.edu; zank@bartol.udel.edu)

S. Oughton, Department of Mathematical and Computational Sciences, University of St. Andrews, Scotland, UK KY16 9SS

Y. Zhou, Institute for Computer Applications in Science and Engineering, Langley Research Center, MS 132C Hampton VA 23681

(Received April 29, 1994; revised August 29, 1994; accepted August 29, 1994.) 